\title{
Unusual Cause of Multiple Cranial Nerve Palsy
}

\section{SHAHRIAR MAHBUB, ${ }^{1}$ ABDULLAH AL FARUQ, ${ }^{1}$ MOHAMMAD ASHIK IMRAN KHAN, ${ }^{2}$ HAM NAZMUL AHASAN ${ }^{3}$}


Fig. 1 MRI T1 axial (A) \& coronal (B) section of brain showing lesion extending into left para-sellar area, left sphenoidal sinus, left orbit and pharyngeal space. Left middle ear and mastoid region showing inflammatory changes

A 55 year old male presented with 2 months history of difficulty in deglutition, dimness of vision in his left eye, headache associated with nausea. On examination, a swelling was noted on the hard palate and left posterior cervical lymph nodes were palpable. Cranial nerve examination revealed the left II, III, IV, V, VI, IX, X, XII nerves were affected. Fundoscopy showed left primary optic atrophy.

MRI of brain showed hypointense on T1 and hyperintense lesion on T2 involving the pharyngeal space, extending into left para-sellar, left sphenoidal sinus, left orbit causing mild proptosis (Figure 1). Gadolinium contrast imaging showed lesion in masticator space with irregular rim enhancement. The clinical and imaging features were suggestive of rhabdomyosarcoma. Differentials were nasopharyngeal carcinoma, lymphoma, poorly differentiated carcinoma, metastatic tumor and melanoma. The patient refused further investigation and sought discharge against medical advice.

Rhabdomyosarcoma (RMS) is very uncommon in adult population, being more common in children. The primary sites of RMS are: head and neck (40\%), genitourinary system
(20\%), extremities (20\%), trunk (10\%), and other (10\%). ${ }^{1} \mathrm{RMS}$ in the region of head and neck involves orbit, paranasal sinuses, nasopharynx, nasal cavity, middle ear and soft tissues of the neck. Involvement of these parameningeal regions leads to multiple cranial nerve palsy. It arises from skeletal muscle as well as mastoid air cells and paranasal sinuses. ${ }^{2}$ Local invasion, local recurrence, hematogenous and lymphatic metastasis are common in RMS. Treatment options of RMS include combination of chemotherapy, radiotherapy and surgery depending on the primary tumor site, pathologic findings, and the clinical stage of the tumor.

\section{Conflict of Interest: None}

\section{References:}

1. Moon HS, Kwon SW, Lee JH. A case of alveolar rhabdomyosarcoma of the ethmoid sinus invading the orbit in an adult. Korean J Ophthalmol 2006;20:70-75

2. Koenigsberg RA, Noah R, Turtz A, et al. Rhabdomyosarcoma of the paranasal sinuses in an adult. Clinical Imaging 1995;19:234-236

1. FCPS (Medicine) course student, Department of Medicine, Dhaka Medical College

2. Post-graduate trainee, Department of Medicine, Dhaka Medical College

3. Professor, Department of Medicine, Dhaka Medical College

Correspondence: Dr. Md. Shahriar Mahbub, Department of Medicine, Dhaka Medical College, Dhaka, Bangladesh. E-mail: shahriar04@yahoo.com 